\title{
Sensitivity to a Phytotoxin from Rhizoctonia solani Correlates with Sheath Blight Susceptibility in Rice
}

\author{
Steven A. Brooks \\ U.S. Department of Agriculture-Agricultural Research Service, Dale Bumpers National Rice Research Center, 2890 Highway 130 East, \\ Stuttgart, AR 72160. \\ Accepted for publication 23 April 2007.
}

\begin{abstract}
Brooks, S. A. 2007. Sensitivity to a phytotoxin from Rhizoctonia solani correlates with sheath blight susceptibility in rice. Phytopathology 97: 1207-1212.

Sheath blight is one of the most important and intractable diseases of
rice (Oryza sativa) where limited control has been achieved using
traditional approaches. Quantitative inheritance, extraneous traits, and
environmental factors confound genetic analysis of host resistance. A
method was developed to isolate and utilize a phytotoxin from Rhizoc-
tonia solani to investigate the genetics of sheath blight susceptibility.
Infiltration of the toxin preparation into plant leaves induced necrosis in
rice, maize, and tomato. Using 17 rice cultivars known to vary in sheath

blight resistance, genotypes were identified that were sensitive (tox-S) and insensitive (tox-I) to the toxin, and a correlation $(r=0.66)$ between toxin sensitivity and disease susceptibility was observed. Given the broad host range of $R$. solani, genotypes of host species may be both tox-S and tox-I. A total of $154 \mathrm{~F}_{2}$ progeny from a cross between Cypress (tox-S) and Jasmine 85 (tox-I) segregated in a 9:7 ratio for tox-S/tox-I, indicating an epistatic interaction between two genes controls sensitivity to the toxin in rice. This work provides the means to genetically map toxin sensitivity genes and eliminate susceptible genotypes when developing sheath blight-resistant rice cultivars.
\end{abstract}

Sheath blight is one of the most economically important diseases of cultivated rice (Oryza sativa L.) worldwide. The disease occurs in most rice-producing areas and is second in importance only to rice blast (20). The causal agent is the necrotrophic fungus Rhizoctonia solani Kühn (teleomorph Thanatephorus cucumeris (A. B. Frank) Donk) anastomosis group (AG) 1 , intraspecific group IA (20). $R$. solani is soilborne and survives in the absence of a host as asexually produced sclerotia. Sclerotia can survive in the soil for 2 years, accumulate over time, and are spread quickly during field preparation and flood application (20). Mature sclerotia are buoyant and collect around rice plant culms with the application of permanent flood. Initial infections occur at the waterline as water soaked lesions on leaf sheaths. Subsequent infections are caused by runner hyphae that move upward to aerial portions of the plant, produce additional lesions, and complete the disease cycle by producing sclerotia on the surfaces of leaves and leaf sheaths (20). The disease develops rapidly at flowering when the canopy is most dense, creating a microclimate favorable to fungal growth and proliferation. High yielding, susceptible, semi-dwarf cultivars are particularly vulnerable to sheath blight as short stature, high tillering, dense planting, and high nitrogen input create conditions suitable for disease epidemics (15). In the southern United States, long grain japonica type cultivars that have little resistance to sheath blight can have yield losses as high as $44 \%$ (14).

Management practices have had little impact on sheath blight incidence, with foliar applications of fungicide being the most effective control strategy (6). The broad host range of $R$. solani

Corresponding author: S. A. Brooks; E-mail address: ricegenes@mac.com

doi:10.1094/PHYTO-97-10-1207

This article is in the public domain and not copyrightable. It may be freely reprinted with customary crediting of the source. The American Phytopathological Society, 2007.
Additional keywords: disease resistance, host-selective toxin. and long-term survival of sclerotia preclude the use of rotation or fallow as measures to reduce amounts of primary inoculum. Furthermore, no source of complete genetic resistance is known in $O$. sativa or its wild relative species (15). Rice cultivars vary in levels of tolerance to the disease, where tolerance is often conferred or confounded by extraneous factors such as plant height or maturity $(15,16)$. The development of resistant rice cultivars for the southern United States has promise for reducing disease severity and corresponding yield losses. However, current methods for identifying resistance genes are inadequate for resolving complex quantitative inheritance, confounding traits, and environmental influence.

Production of host-selective toxins (HSTs) by $R$. solani is known and is consistent with the necrotrophic nature of the pathogen $(1,5,11,13,18,19)$. HSTs can increase the virulence of a pathogen (e.g., HC-toxin on maize) or are often ("pathogenicity determinants") required for a pathogen to incite disease (reviewed in 23). Previous research has shown that phenyl acetic acid (PAA) $(11,13)$, derivatives of PAA (13), a phenolic compound (18), and a carbohydrate (19) acted as phytotoxins of $R$. solani. Given the broad host range and diversity within the $R$. solani species (3), it is not surprising that multiple phytotoxins have been reported for this fungus. PAA has been the most widely described in the literature $(1,5,11,13)$ and is known as a virulence factor for AG3 on potato (11). PAA production has been reported from isolates recovered from diseased rice plants (5), but previously has been shown not to induce necrosis of plant tissue (1), and more recently not to be the primary toxin involved in rice sheath blight (19). Although the structure of the $R$. solani AG1-IA toxin is unknown (19), phytotoxin sensitivity remains an attractive target for unraveling the complex inheritance of resistance to sheath blight in rice. The designation phytotoxin is used in this work instead of HST, because the $R$. solani toxin described here has multiple host and genotype specificities. The objectives of this study were to develop an assay for cultivar response to the $R$. solani phytotoxin, determine the correlation between toxin sensitivity 
and disease susceptibility, and resolve the genetic control of toxin sensitivity in rice.

\section{MATERIALS AND METHODS}

Plant material. The University of Arkansas Rice Research and Extension Center (UA RREC, Stuttgart, AR) provided the following rice cultivars: Bengal, Cybonnet, Cypress, Drew, Jasmine 85, Katy, Kaybonnet, L202, Leah, Madison, Mars, Newbonnet, and soybean cv. Pioneer 94M80. Rice cv. Cocodrie and rice germplasm MCR01-0277 were provided by the Genetic Stocks Oryza Collection (USDA-ARS, Dale Bumpers National Rice Research Center, Stuttgart, AR). S. Pinson (USDA-ARS, Beaumont, TX) provided rice cvs. Lemont and Teqing. Rice Tec (Alvin, TX) provided rice hybrid XP710. The Wheat Genetics Resource Center (Kansas State University, Manhattan, KS) provided wheat cv. Chinese Spring and barley cv. Morex. Arabidopsis stock Col-0 was obtained from the Arabidopsis Biological Resource Center (Ohio State University, Columbus, OH). Maize inbred line B73 was obtained from the North Central Regional Plant Introduction Station (USDA-ARS, Ames, IA). Seed of tomato cv. Red Cherry was purchased at a local garden center (Ferry-Morse Seed Co., Fulton, $\mathrm{KY}$ ). A population of $154 \mathrm{~F}_{2}$ progeny derived from a cross of rice cvs. Cypress (very susceptible [VS], tox-S) and Jasmine 85 (moderately resistant [MR], tox-I) was developed to determine the inheritance of toxin sensitivity.

Fungal isolates. $R$. solani isolate B1 was collected from naturally infected rice plants growing in plots located at UA RREC in 2004. Sclerotia collected from diseased plants were surface-sterilized for $5 \mathrm{~min}$ in $0.6 \%$ sodium hypochlorite, blotted dry on sterile paper towels, and transferred to water agar in plates. Fungal hyphae were transferred twice onto water agar and finally onto potato dextrose agar (PDA). The PDA culture was grown at $28^{\circ} \mathrm{C}$ in constant light until sclerotia formed. The culture and medium were then dried completely, and the desiccated isolate was stored at room temperature until needed.

Isolate B1 was confirmed as R. solani by Koch's postulates and DNA-based molecular markers. A sclerotium from the original PDA plate was transferred to a new PDA plate and grown for 2 days at $28^{\circ} \mathrm{C}$ with constant light. Plugs of PDA from the leading edge of the colony were removed from the plate with the large end of a sterile Pasteur pipette. PDA plugs were transferred to the base of seedlings of cv. Cypress (VS) where fungal infection reproduced the water-soaked lesions characteristic of sheath blight symptoms. Koch's postulates were completed by reculture of the fungus from inoculated plants. Cultures of isolate B1 showed characteristic hyphal morphology by microscopic examination. In addition, a PDA plug was removed to inoculate a liquid culture of potato dextrose broth (PDB). Fifty milliliters of PDB was grown for 7 days at $28^{\circ} \mathrm{C}$ with constant light and the mycelial mass was recovered by filtration through Miracloth (Calbiochem, La Jolla, $\mathrm{CA}$ ) and washed with sterile water. DNA was purified by a CTAB extraction protocol (2). Isolate B1 was screened with speciesspecific molecular markers for the rDNA internal transcribed spacer (ITS) region (9), confirmed as $R$. solani, and assigned to AG1-IA by sequence alignment of the rDNA ITS region (10).

Fungal culture and toxin recovery for rice cultivar screening. Rice leaf medium (RLM) was used to grow liquid cultures of $R$. solani for toxin isolation and rice cultivar screening. On a small scale, RLM was prepared by adding $4 \mathrm{~g}$ of leaf tissue (cv. Cypress), cut in pieces approximately $2 \mathrm{~cm}$ long, to $100 \mathrm{ml}$ of distilled water in a 500-ml Erlenmeyer flask. The flask was covered with aluminum foil and autoclaved for $35 \mathrm{~min}$ at $1.03 \mathrm{bar}$ and $121^{\circ} \mathrm{C}$. A PDA plug of $R$. solani, obtained as described previously, was used to inoculate the RLM. The medium was incubated at $28^{\circ} \mathrm{C}$ with constant light for 14 days. To avoid any batch-to-batch variation in toxin concentration, a single 1-liter batch of toxin was prepared for all plant screening. From the 1-liter starting volume, $880 \mathrm{ml}$ was recovered (post-autoclave and 14 days of growth) and concentrated 10 -fold to $88 \mathrm{ml}$ (10× TOX), which was sufficient for 880 infiltrations at $0.1 \mathrm{ml}$ of $10 \times$ TOX per replicate.

The liquid fraction from 14-day-old cultures of $R$. solani in RLM was recovered by filtration through Whatman 541 filter paper (Whatman International Ltd., Maidstone, England) in a Büchner funnel under vacuum. The medium was stored overnight at $-20^{\circ} \mathrm{C}$ in $250-\mathrm{ml}$ bottles, thawed at $4^{\circ} \mathrm{C}$, and then centrifuged at $4,000 \times g$ for $10 \mathrm{~min}$ at $4^{\circ} \mathrm{C}$ to remove the precipitate. The medium was then concentrated to 10 times the original concentration $(10 \times \mathrm{TOX})$ in-vacuo at $50^{\circ} \mathrm{C}$ with a Rotavapor R-205 (BÜCHI Labortechnik AG, Postfach, Switzerland). One-milliliter aliquots of $10 \times \mathrm{TOX}$ were stored at $-20^{\circ} \mathrm{C}$ prior to use. Aliquots thawed to room temperature were centrifuged at $10,000 \mathrm{rpm}$ for 2 min to pellet the precipitate formed during freezing. The supernatant was removed for infiltration into rice leaves.

One milliliter of $10 \times$ TOX was loaded into a 1-cc syringe and connected to a Hagborg device (7). One hundred microliters of $10 \times$ TOX was infiltrated into rice leaves per replicate on 45- to 60 -day-old plants. A minimum of 10 replications per rice cultivar were evaluated. Toxin-treated leaves were scored 3 to 5 days postinfiltration for necrosis. Water and uninoculated medium (10x, prepared in the same manner as toxin samples) were used as negative controls.

Sensitivity of rice cultivars to toxin preparations was measured as a percentage of necrosis in the infiltrated leaf area. Digital images were taken of the initial toxin infiltrations (water soaked spots) with a C-8080 digital camera (Olympus Corp., Tokyo, Japan), in super macro mode, from a fixed camera mount. Images were taken again of the necrosis on each leaf, and control images of graph paper were used to standardize subsequent measurements. Digital images were imported into APS Assess software (Lakhdar Lamari, Winnipeg, Canada) for quantification of infiltrated and necrotic leaf areas. These areas were represented as the number of pixels, and a sensitivity rating was calculated as the percent necrosis. The value range was $0 \%$ (no response) to $157 \%$ (fully sensitive), where values $>100 \%$ indicate necrosis in adjacent uninfiltrated tissue.

Numerical values of disease reactions ( 0 to 9 for sheath blight, [16]) were tested for correlation to percent necrosis using the CORR procedure in SAS 9.1.3 (SAS Institute, Cary, NC). The breadth of the rating scale began at 0 (immune or no infection) and extended to 9 (dead plant). Values were assigned as a percentage of disease progress from the waterline vertically to aerial portions of the plant (0 to $100 \%$ infection, F. N. Lee personal communication). Five cultivar reactions are assigned based on the rating scale: resistant (1 to 2 ), moderately resistant (3 to 4 ), moderately susceptible (4 to 5 ), susceptible (5 to 6 ), and very susceptible ( 7 to 8 ).

To check for batch-to-batch consistency in toxin preparations, a bioassay was used to determine relative toxin concentration per sample. A dilution series of $10 \times$ TOX was tested with the method described above to determine the minimum concentration required to induce necrosis (usually $5 \times$ ). Experiments were then performed by using a concentration twice the minimum for biological activity (usually 10x). Toxin preparations that required greater than $5 \times$ minimum concentration for activity were discarded.

Fungal culture and toxin preparation for a seedling assay. Rice hull medium (RHM) was used to prepare toxin samples for leaf painting assays on rice seedlings at the four-leaf stage. RHM was prepared with $30 \mathrm{~g}$ of rice hulls (palea and lemma, obtained from the UA RREC granary) and $10 \mathrm{~g}$ of rough paddy rice (cv. Bengal). The mixture was soaked in deionized water in a 1-liter beaker for $15 \mathrm{~min}$, transferred to a strainer, and rinsed thoroughly. The rinse was used to remove soil and any chemical residues from the field and was complete when the water ran clear. The drained 
mixture was then added to a 1-liter Erlenmeyer flask and autoclaved for $35 \mathrm{~min}$ at $1.03 \mathrm{bar}$ and $121^{\circ} \mathrm{C}$. No additional water was added. The sterile mixture was inoculated with a PDA plug as described above and stored at $28^{\circ} \mathrm{C}$ for 14 days in constant light.

The 14-day-old RHM culture was transferred to a $150-\mathrm{mm}$ Büchner funnel lined with a prewetted piece of Whatman No. 4 filter paper. Two hundred and fifty milliliters of deionized water was added to the culture and mixed with a glass rod to disrupt any large clumps. After $3 \mathrm{~min}$, the mycelium was filtered and reextracted for $3 \mathrm{~min}$ with the filtrate. The mycelium was extracted a total of four times with this method. The toxin solution was then dispensed into $50-\mathrm{ml}$ conical tubes, stored at $-20^{\circ} \mathrm{C}$ overnight, and centrifuged to remove the precipitate. The volume of the toxin solution was then recorded and concentrated to 1/10th volume $(10 \times$ TOX) in-vacuo as described previously. The $10 \times$ TOX was dispensed into 1.5-ml Eppendorf tubes (Brinkman Instruments Inc., Westbury, NY) and evaporated to dryness invacuo at $50^{\circ} \mathrm{C}$ in a Centrivap Concentrator (Labconco, Kansas City, MO). The dried samples were stored at room temperature. Toxin samples were resuspended to $50 \times$ concentration (from original starting volume) with a solution of $0.5 \%$ Valent X-77 spreader (Valent U.S.A., Walnut Creek, CA) in deionized water.

With a cotton swab, 50× TOX was painted on leaf 3 (as counted from lowest leaf) of rice seedlings at the four-leaf stage. Approximately $2 \mathrm{~cm}$ of leaf length was painted, and 10 plants/leaves were treated per cultivar. Trays of treated seedlings were misted lightly with water, covered with a humidome (Acrodome, Acro Plastics Ltd., Edmonton Canada), and transferred to a $30^{\circ} \mathrm{C}$ growth chamber for $48 \mathrm{~h}$ in constant light. Then, trays were transferred to the greenhouse with humidomes removed for an additional 3 days. Phenotype was scored visually as sensitive or insensitive $(+,-)$ by the presence or absence of necrotic spots in the painted leaf area.

\section{RESULTS}

Toxin sensitivity testing media. Two liquid media were developed for culture of $R$. solani and phytotoxin extraction in this study. Each medium was prepared to address specific problems in methodology development. In general, rich media damaged leaf tissue when infiltrated using the methodology described below. Richard's medium, Fries medium, and PDB all supported excellent fungal growth. However, the damage to leaf tissue from the media alone (negative controls) was substantial, and the ability to distinguish actual necrosis was abolished. In these cases, it is not known if the fungus produced toxin in each culture medium. To overcome this problem, a series of minimal media were developed that would not damage the plants and support fungal growth.

The two new media described herein were found to favor fungal growth, toxin production and did not cause damage as negative controls. RLM was developed for plant screening and found to promote the highest levels of toxin production. Toxin concentration is described relative to the volume of the original medium (see bioassay). RLM did not damage plants up to $50 \times$ concentration, and RLM-produced toxin was active as low as $5 \times$ concentration with typical leaf assays performed at $10 \times$ (coined $10 \times$ TOX). Cultures were grown to an optimum time of 10 to 14 days. Toxin production did not increase after 10 days. At this time, a dense mat of mycelia covered the culture surface and sclerotia had started to form.

RHM was developed for a seedling assay. In this assay, volumes as low as $5 \mu$ were used to paint a seedling leaf. For this reason, a concentrated toxin preparation was required, because the volume to area ratio was low $(50 \times$ was used). At $50 \times$ concentration, a medium was needed that did not damage the seedlings. At this concentration, RLM alone damaged leaves. RHM did not damage leaves up to $100 \times$ concentration.
Toxin sensitivity bioassay. RLM-produced toxin (10× TOX) was used for all plant assays. The method described above was nondestructive to rice plants. Toxin was infiltrated into the leaves of intact plants (field or greenhouse) with a Hagborg device and did not cause any systemic symptoms. Necrosis was limited to the treated area in sensitive cultivars (Cypress, Fig. 1A and B), and numerous replications could be made on a single plant (two replicates per leaf, all fully extended leaves of the plant). No necrosis was observed in insensitive cultivars (Jasmine 85, Fig. 1C and D). The toxin assay was performed on plants as young as 30 days and as late as heading, but the best results were obtained on 45- to 60 -day-old plants. At this stage, plants were sufficiently developed so mechanical damage rarely occurred, and toxin readily penetrated the leaf. Replicate infiltrations were performed under different conditions as follows: (i) multiple simultaneous infiltrations, (ii) repetition over time, and (iii) on ratooned plants. In all replications, visual ratings were consistent, indicating the host response to toxin infiltration does not affect the response to subsequent infiltrations. Therefore, replication of the response to the toxin is unlimited on a single clonally propagated plant.

Supplemental seedling assay. The Hagborg device used in the standard assay caused mechanical damage that was too severe to perform the test on young plants ( $<30$ days old). To develop a seedling assay, a series of chemical surfactants were tested for toxin spreading on leaf surfaces (Latron CS-7, Rohm and Haas, Philadelphia, PA; Tween 20, Sigma Chemical Co., St. Louis, MO; and Silwet L-77, General Electric Silicones, Friendly, WV; not shown). In the absence of a surfactant, the toxin solution did not adhere to the cuticle and rolled off the leaf. Valent X-77 (Valent U.S.A. Corp.) provided the most effective combination of spreading ability and lack of leaf damage (as a negative control). The spreading ability of X-77 allowed rapid drying of the toxin on the surface of the leaf, and consequently, no effect of the toxin was observed. It was necessary to cover plants for 2 days with a humidome and maintain near-100\% humidity to obtain consistent results. These conditions promoted surfactant-aided penetration of the toxin into leaf tissues. With this assay, $50 \times$ concentrated RHM toxin in $0.5 \% \mathrm{X}-77$ reproduced the diagnostic necrotic spots seen in the standard assay (not shown).

Bioassay for toxin concentration. For consistency, all on-plant assays were performed from a single large batch of toxin (prepared from RLM or RHM). In addition, a bioassay was used to determine consistency between toxin preparations in the development of toxin sensitivity assays. Generally, $5 \times$ concentrated culture filtrate was the minimum required to induce necrosis in rice leaves, and $10 \times$ toxin was used for evaluating rice plants. On average, $10 \times$ toxin contained $15.7 \mathrm{mg}$ of lyophilized culture filtrate per $\mathrm{ml}$ of water. In evaluating a series of cultivars (described below), the same results were obtained if $5 \times, 10 \times$, or $20 \times$ RLMproduced toxin was used. Therefore, the bioassay was sufficient for approximate toxin concentration given the tolerances found for consistent results.

Host selectivity of toxin. Arabidopsis (Col-0), tomato (Red Cherry), soybean (Pioneer 94M80), wheat (Chinese Spring), barley (Morex), and maize (B73) were tested for sensitivity to the $R$. solani toxin in addition to rice (not shown). Tomato and maize were the only species that were sensitive to $10 \times$ TOX and expressed the same necrotic phenotype seen in rice. To determine if the lack of response in the other species was due to a low toxin concentration, a series of increases in RLM-produced toxin concentration were applied along with RLM as a negative control. In all experiments, the plants remained insensitive to RLM-produced toxin (Arabidopsis at 50x; wheat, barley, and soybean at 20x). These represent the maximum concentrations for each species where the negative controls did not damage leaf tissue.

Differential reaction of rice cultivars to toxin. Genotype specific response to the $R$. solani toxin was observed by a differential response of rice cultivars to the toxin. Figure 2 summarizes 
the results of toxin sensitivity in 17 rice cultivars. Five cultivars (Cocodrie, Cypress, L202, Lemont, and Madison) were rated as fully sensitive to the toxin based on $\approx 100 \%$ average necrosis in the infiltrated leaf area. The significant variation in measurement,

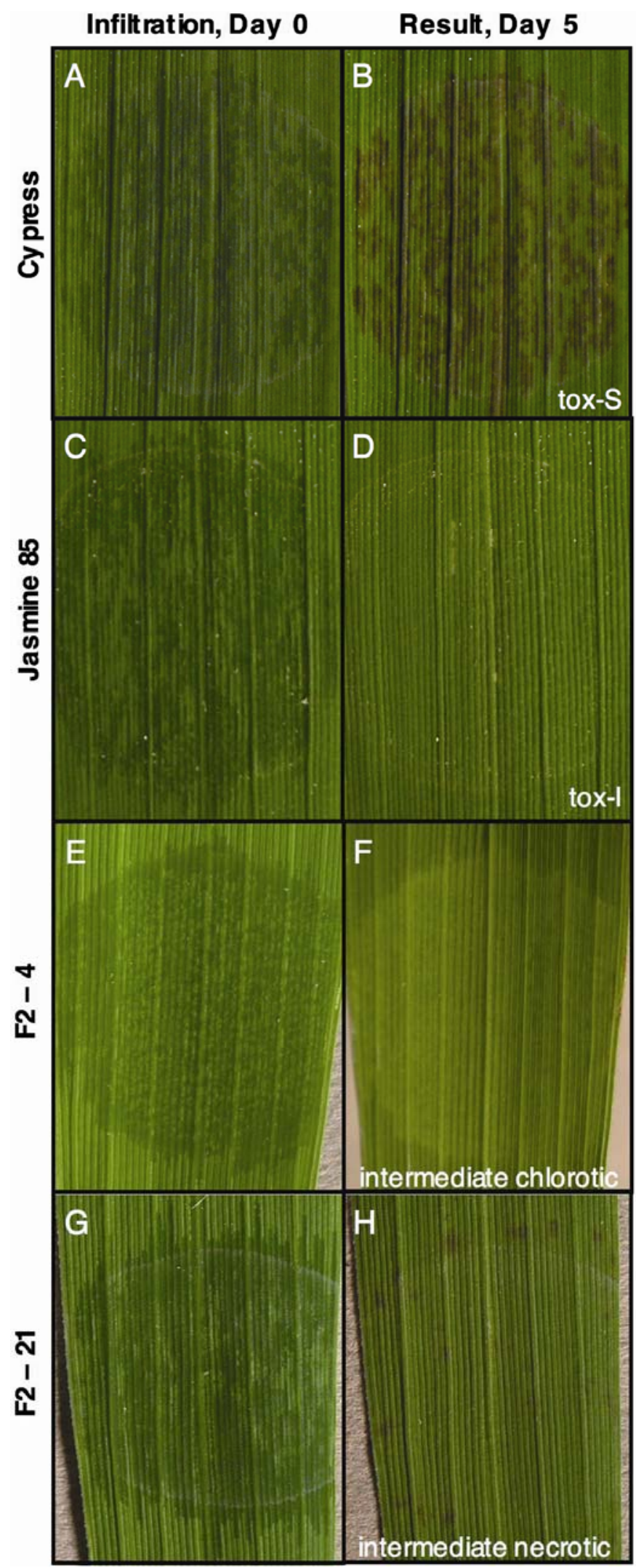

Fig. 1. Infiltration of the Rhizoctonia solani phytotoxin into the leaves of rice cultivars A, Cypress, C, Jasmine 85,E, F2 - 4, and G, F2 - 21. Reaction to the toxin is $\mathbf{B}$, fully sensitive in Cypress, $\mathbf{D}$, completely insensitive in Jasmine $85, \mathbf{F}$, intermediate chlorotic in F2 - 4, and $\mathbf{H}$, intermediate necrotic in F2 - 21. displayed as standard deviation bars in Figure 2, is the result of limited accuracy in measuring the initial infiltrated area, because the color of the water-soaked infiltrations was not significantly different from the surrounding leaf area (Fig. 1A and C). Small changes to parameters in APS Assess resulted in large changes in detected area. In contrast, APS Assess gave precise measurements of leaf necrosis because of the significant contrast in color. Even with this source of error, it is clear that these cultivars were completely sensitive to the toxin when comparing digital images side by side. For example, necrosis is seen throughout the infiltrated leaf area of Cypress (Fig. 1A and B). Two cultivars (Leah and Teqing) were rated as partially sensitive. These cultivars consistently showed partial necrosis in the treated leaf area. Bengal, Cybonnet, Drew, Jasmine 85, Katy, Kaybonnet, Mars, MCR010277, Newbonnet, and XP710 were rated insensitive to the toxin.

The 17 surveyed cultivars also varied in their level of known field resistance (Fig. 2). Five rated as very susceptible (VS), one susceptible (S), six moderately susceptible (MS), and five moderately resistant (MR). These ratings cover the rating spectrum for sheath blight resistance. With one exception (Cybonnet VS, toxI), the VS and S rated cultivars were fully sensitive to the toxin, and the more resistant cultivars (MS, MR) possessed partial or complete insensitivity to the toxin. A significant positive correlation is present between disease severity (based on numerical values for sheath blight disease ratings) and percent necrosis in the 17 cultivars $(r=0.66, P=0.0039)$.

Genetic regulation of toxin sensitivity. A Cypress (VS, tox$\mathrm{S}$ )/Jasmine 85 (MR, tox-I) $\mathrm{F}_{2}$ population was used to determine the inheritance of toxin sensitivity in these cultivars. First, four individual $F_{1}$ plants were found to be fully sensitive to the toxin and displayed the same phenotype as the sensitive parent Cypress (not shown). Thus, sensitivity to the toxin is dominant in this cross. Second, $154 \mathrm{~F}_{2} \mathrm{~s}$ were screened with the toxin with at least three replications per plant. In these progeny, two nonparental (intermediate) phenotypes were identified. One phenotype produced chlorosis in the infiltrated area (Fig. 1E and F, F2 - 4), the other produced minor necrotic flecks (Fig. $1 \mathrm{G}$ and H, F2 - 21). These phenotypes were so close to insensitivity that they could not readily be distinguished from the tox-I type and were collectively scored as tox-I. This contrasts the dramatic difference in toxin sensitivity present in the parents of the cross (Figs. 1 and 2). The resulting phenotypic data showed an excess of insensitive types and did not fit the expected 3:1 ratio for a single dominant gene. The presence of two intermediate phenotypes prompted a test for two genes segregating in the population. Since intermediate phenotypes could not be distinguished from insensitive phenotypes, the data could not be tested for 9:3:3:1 segregation. Instead, the data were tested for fit to a 9:7 ratio of two epistatic genes ( 87 sensitive and 67 insensitive observed, 86.6 sensitive and 67.4 insensitive expected). The critical value of chi-square ( $\mathrm{df}=$ 1 , alpha $=0.05$ ) is 3.841 . The calculated chi-square was 0.004 , and failed to reject the null hypothesis for two epistatic genes.

\section{DISCUSSION}

Sheath blight is one of the most important diseases of cultivated rice worldwide, and its importance is expected to increase due to cultivation of susceptible cultivars and agricultural practices that are conducive to disease (20). The high degree of phenotypic variability and quantitative inheritance from known sources of resistance (16) has hindered progress toward understanding sheath blight resistance and incorporating resistance genes into elite cultivated germplasm. To date, no genes have been identified for sheath blight resistance, nor are molecular markers available for marker-assisted selection of known quantitative trait loci (QTL). Disease assays have been highly variable and are often unreliable for providing precise phenotypic data needed in genetic mapping. To this end, this study was conducted to determine the inheritance 
of toxin sensitivity and the association of toxin reaction with sheath blight resistance.

In the past 40 years, a modest amount of work has been done with the host-selective toxins produced by $R$. solani $(1,5,11$, 13,18,19). These efforts focused primarily on the toxin itself and did not report any methods to use an HST for genetic analysis or resistance selection. Mandava et al. (13) suggested that a potential utility of the HST was to identify resistance to $R$. solani, but no method has been developed or applied. Several methods have been used to bioassay a response to HST fractions in rice (19), but all are lacking in throughput and efficiency for population phenotyping. Here, a reliable and efficient toxin assay is described that is appropriate for genetic analysis and breeding. In addition, a supplemental seedling assay is described that allows data collection to begin more than 1 month ahead of the standard assay. Its value is in eliminating toxin-sensitive genotypes early in the screening process.

Vidhyasekaran et al. (19) described the partial purification and characterization of an HST (coined RS-toxin) from $R$. solani isolated from rice in India. Here, the "RS-toxin" designation is not used because some of the current results contrast with this earlier work, which may be due to variation in isolates collected from different countries. In a survey of rice cultivars and additional plant species, Vidhyasekaran et al. (19) identified hosts and nonhosts by reaction to the fungal pathogen. By using an electrolyte leakage assay on RS-toxin infiltrated tissue of identified hosts (rice and cotton) and nonhosts (sapota and coconut), the authors concluded that RS-toxin shares the same host specificity as the pathogen and is therefore an HST. It is unclear how many varieties of each of the four species were tested. However, the authors were only able to demonstrate host specificity by comparing host and nonhosts, not by variability within a species.

The work here adds to that of Vidhyasekaran et al. (19) in that it identified insensitivity to this phytotoxin in wheat, barley, soybean, and Arabidopsis. The insensitivity found in these species may be due to the specific genotype of the species tested. In rice, multiple distinct phenotypes were observed, ranging from completely sensitive to insensitive cultivars. Therefore, generalizing the reaction of a species to a toxin may be invalid if only a single cultivar is used. In addition, the $R$. solani species complex consists of at least 13 AGs, with host ranges that include all major crop species (3). It is possible that different AGs, or subgroups within a single AG, produce different phytotoxins. For example, Mandava et al. (13) identified PAA and PAA-derivatives as phytotoxins of $R$. solani AG-IV isolated from soybean. However, Vidhyasekaran et al. (19) showed that RS-toxin was not PAA. Furthermore, a bioassay and high performance liquid chromatography (HPLC) showed that PAA did not cause necrosis in tox$S$ genotypes of rice and had a lower molecular weight than the phytotoxin found in 10 different isolates of $R$. solani AG 1-IA from Arkansas (S. A. Brooks, unpublished data). These isolates likely produce the same toxin as determined by HPLC and bioassay on rice cultivar differentials, but the toxin is not PAA (S. A. Brooks, unpublished data). At this time, the structure of the $R$. solani phytotoxin reported herein and its relationship to RStoxin are unknown.

In a survey of 17 rice cultivars, a significant positive correlation was found between toxin sensitivity and disease susceptibility. An absolute correlation was not expected or observed, because field ratings (complicated by unrelated factors) are not necessarily a measure of genetic resistance (20). Similarly, Rahman et al. (17) found variation in field resistance to Cephalosporium stripe within toxin-sensitive genotypes of wheat. It is clear that pathogenicity in $R$. solani is not exclusively dependent upon the toxin as is the case in some other systems (reviewed in 23). This is evident in the very susceptible cv. Cybonnet that was insensitive to the toxin. It is important to note that semi-dwarf varieties with dense canopies like Cybonnet create a microclimate that favors disease development (15). Since susceptibility is known in the Cybonnet pedigree, field ratings may be incapable of discerning the effect of the tox-I phenotype. Also, cvs. Leah and Teqing show an apparent disparity between disease (MR) and toxin (partially sensitive) ratings. However, QTL mapping (16) has shown that a major resistance QTL from Teqing corresponds to tall plant stature and late maturity, where both characters are known to skew sheath blight ratings toward resistance $(16,20)$. In the case of Teqing, the MR rating is bolstered by tall-late traits, which confound actual genetic resistance. Given the limits of field disease ratings, more accurate disease assays (8) are needed to test near isogenic lines that differ in toxin reactions to precisely quantify the effect of toxin sensitivity genes on resistance.

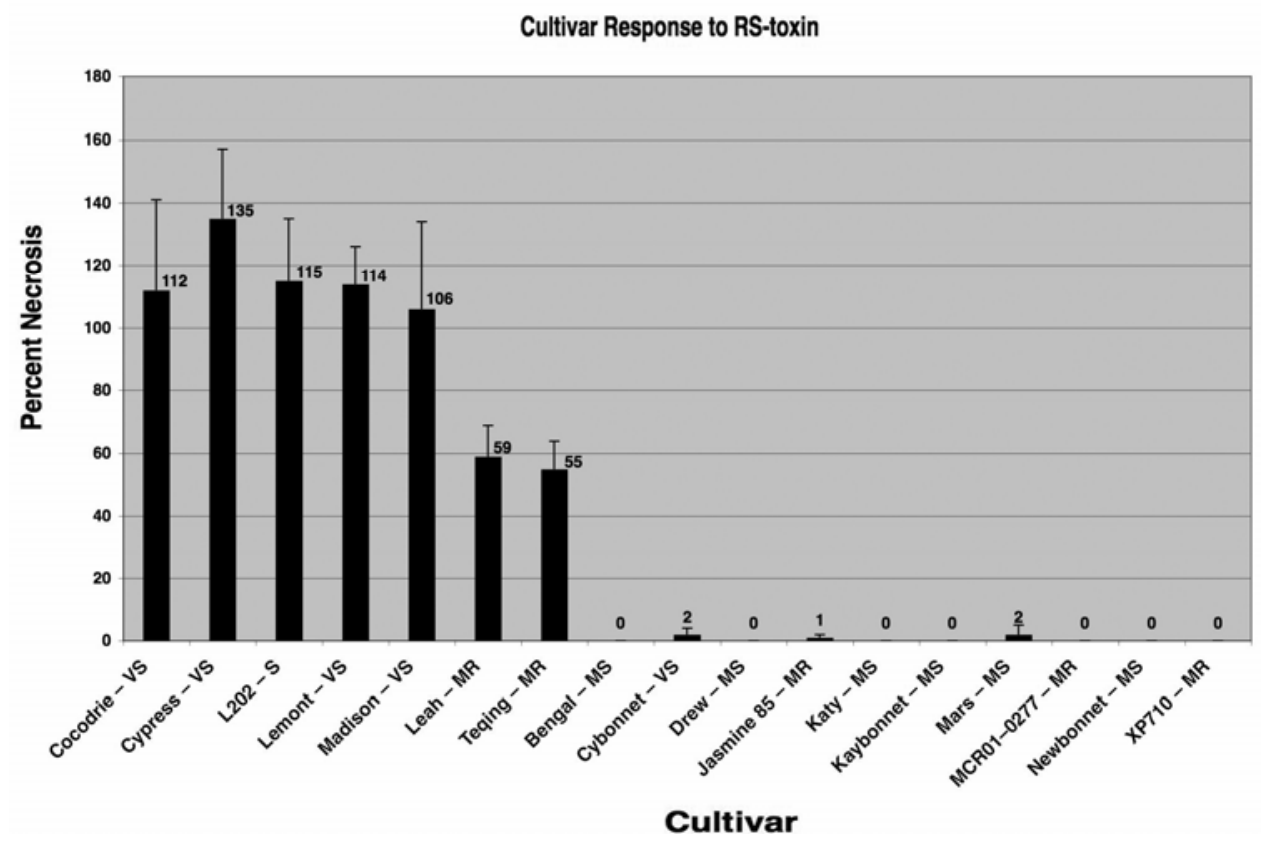

Fig. 2. Summary of necrotic response to the Rhizoctonia solani phytotoxin in 17 rice cultivars. Average percent necrosis for each cultivar is shown with standard deviation bars. Sheath blight disease ratings are provided by each cultivar name $(4,14,15,21,22,24)$. 
In this study, sensitivity to the phytotoxin was conferred by two dominant genes, but the allelic condition of the parents of the mapping population remains in question. Since single plant selections of inbred cultivars were used as parents, it is likely that each plant was homozygous at both loci. For illustrative purposes herein, the loci have been labeled ' $N$ ' for necrosis and ' $C$ ' for chlorosis. Since Cypress is fully sensitive and Jasmine 85 completely insensitive, it appears that the cross was NNCC $\times$ nncc (not NNcc $\times$ nnCC). Thus, the fully sensitive $\mathrm{F}_{1} \mathrm{~s}$ are $\mathrm{NnCc}$, and $\mathrm{F}_{2} \mathrm{~S}$ segregate as $\mathrm{NxCx}$ (fully sensitive), $\mathrm{nnCx}$ (intermediate chlorotic), Nxcc (intermediate necrotic), and nncc (completely insensitive).

Independent inheritance of necrosis and chlorosis is also observed in the wheat-tan spot interaction (12). The typical tan spot phenotype consists of necrotic lesions surrounded by obvious chlorotic halos. This contrasts sheath blight symptoms of rice, which have disease lesions bordered by a narrow necrotic margin. In the analysis of response to the $R$. solani toxin, chlorosis was only observed in segregating progeny of a mapping population. These segregants showed chlorosis throughout the infiltrated area. Apparently, when dominant alleles are present at both loci, complete necrosis occurs, masking the chlorotic phenotype. It appears that the gene that mediates chlorosis is a necessary prelude for complete necrosis, because a dominant allele at the necrotic locus alone is not sufficient for complete necrosis, and only a minor necrotic fleck phenotype is observed. This phenotypic relationship is consistent with the genetic model proposed and suggests a role for two genes regulating $R$. solani toxin sensitivity in rice.

Here, an assay is presented for determining the response of rice plants to the phytotoxin produced by $R$. solani. It is clear that sensitivity to the toxin is not the only requirement for susceptibility, although a correlation has been observed between the two phenotypes. Additional research is needed to determine the contribution of the toxin to pathogen virulence. To this end, backcrossing has begun to introduce both dominant alleles ( $\mathrm{N}$ and $\mathrm{C}$ ) into the Jasmine 85 (MR) background, to assay for a reduction in the level of resistance in near isogenic progeny.

This study has provided the means to map the sensitivity genes, and a map-based cloning strategy is underway to identify each gene. This strategy is an advancement toward mapping sheath blight resistance genes that previously were scored as QTL. This work describes a precise phenotyping method, genetic inheritance of the trait, the means to eliminate toxin-sensitive genotypes from germplasm, and a demonstration that toxin sensitivity is correlated with susceptibility to this important disease.

\section{ACKNOWLEDGMENTS}

This work was supported in part by the USDA Cooperative State Research, Education and Extension Service - National Research Initiative - Applied Plant Genomics Program entitled "RiceCAP: A coordinated research, education, and extension project for the application of genomic discoveries to improve rice in the United States" (USDA/ CSREES grant 2004-35317-14867). I thank the following people for contributions to this work: M. Anders, W. Bockus, R. Bryant, J. Gibbons, A. Jackson, Y. Jia, F. Lee, J. Leslie, K. Moldenhauer, and K. Yeater. I also would like to recognize A. Jackson and G. Miller for excellent technical support. Mention of a trademark of a proprietary product does not constitute a guarantee or warranty of the product by the U.S. Department of Agriculture, and does not imply its approval to the exclusion of other products that may also be suitable.

\section{LITERATURE CITED}

1. Aoki, H., Sassa, T., and Tamura, T. 1963. Phytotoxic metabolites of Rhizoctonia solani. Nature 200:575.

2. Brooks, S. A., Huang, L., Herbel, M. N., Gill, B. S., Brown-Guedira, G., and Fellers, J. P. 2006. Structural variation and evolution of a defensegene cluster in natural populations of Aegilops tauschii. Theor. Appl. Genet. 112:618-626.

3. Carling, D. E., Baird, R. E., Gitaitis, R. D., Brainard, K. A., and Kuninaga, S. 2002. Characterization of AG-13, a newly reported anastomosis group of Rhizoctonia solani. Phytopathology 92:893-899.

4. Chu, Q. R., Linscombe, S. D., Rush, M. C., Groth, D. E., Oard, J., Sha, X., and Utomo, H. S. 2006. Registration of a C/M doubled haploid mapping population of rice. Crop Sci. 46:1417.

5. Danson, J., Wasano, K., and Nose, A. 1999. Effect of culture nutrients on the production of Rhizoctonia solani toxins. IRRN 24:16.

6. Groth, D. E., and Bond, J. A. 2006. Initiation of rice sheath blight epidemics and effect of application timing of azoxystrobin on disease incidence, severity, yield, and milling quality. Plant Dis. 90:1073-1076.

7. Hagborg, W. A. F. 1970. A device for injecting solutions and suspensions into thin leaves of plants. Can. J. Bot. 48:1135-1136.

8. Jia, Y., Correa-Victoria, F., McClung, A., Zhu, L., Liu, G., Wamishe, Y., Xie, J., Marchetti, M. A., Pinson, S. R. M., Rutger, J. N., and Correll, J. C. 2007 Rapid determination of rice cultivar responses to the sheath blight pathogen Rhizoctonia solani using a micro-chamber screening method. Plant Dis. 91:485-489.

9. Johanson, A., Turner, H. C., McKay, G. J., and Brown, A. E. 1998. A PCR-based method to distinguish fungi of the rice sheath-blight complex, Rhizoctonia solani, R. oryzae and R. oryzae-sativae. FEMS Microbiol. Lett. 162:289-294.

10. Kuninaga, S., Natsuaki, T., Takeuchi, T., and Yokosawa, R. 1997. Sequence variation of the rDNA ITS regions within and between anastomosis groups in Rhizoctonia solani. Curr. Genet. 32:237-243.

11. Lakshman, D. K., Liu, C., Mishra, P. K., and Tavantzis, S. 2006. Characterization of the arom gene in Rhizoctonia solani, and transcription patterns under stable and induced hypovirulence conditions. Curr. Genet. 49:166-177.

12. Lamari, L., and Bernier, C. C. 1989. Toxin of Pyrenophora triticirepentis: Host-specificity, significance of disease, and inheritance of host reaction. Phytopathology 79:740-744.

13. Mandava, N. B., Orellana, R. G., Warthen, J. D., Jr., Worley, J. F., Dutky, S. R., Finegold, H., and Weathington, B. C. 1980. Phytotoxins in Rhizoctonia solani: Isolation and biological activity of $m$-hydroxy- and $m$ methoxyphenylacetic acids. J. Agric. Food Chem. 28:71-75.

14. Marchetti, M. A., and Bollich, C. N. 1991. Quantification of the relationship between sheath blight severity and yield loss in rice. Plant Dis. 75:773-775.

15. Pan, X. B., Rush, M. C., Sha, X. Y., Xie, Q. J., Linscombe, S. D., Stetina, S. R., and Oard, J. H. 1999. Major gene, nonallelic sheath blight resistance from the rice cultivars Jasmine 85 and Teqing. Crop Sci. 39:338346.

16. Pinson, S. R. M., Capdevielle, F. M., and Oard, J. H. 2005. Confirming QTLs and finding additional loci conditioning sheath blight resistance in rice using recombinant inbred lines. Crop Sci. 45:503-510.

17. Rahman, M., Mundt, C. C., Wolpert, T. J., and Riera-Lizarazu, O. 2001. Sensitivity of wheat genotypes to a toxic fraction produced by Cephalosporium gramineum and correlation with disease susceptibility. Phytopathology 91:702-707.

18. Sherwood, R. T. 1965. Method of producing a phytotoxin. U.S. patent $3,179,653$

19. Vidhyasekaran, P., Ponmalar, T. R., Samiyappan, R., Velazhahan, R., Vimala, R., Ramanathan, A., Paranidharan, V., and Muthukrishnan, S. 1997. Host-specific toxin production by Rhizoctonia solani, the rice sheath blight pathogen. Phytopathology 87:1258-1263.

20. Webster, R. K., and Gunnell, P. S. 1992. Compendium of Rice Diseases. American Phytopathological Society, St. Paul, MN.

21. Wilson, C. E., Moldenhauer, K., Gibbons, J., Cartwright, R., Lee, F., Norman, R., Bernhardt, J., Blocker, M., Tolbert, A., Taylor, K., Bulloch, J., and Branson, J. 2004. Arkansas Rice Performance Trials, 2002-2004. University of Arkansas Cooperative Extension Service Rice Information Sheet No. 156

22. Wilson, C. E., Moldenhauer, K., Gibbons, J., Cartwright, R., Lee, F., Norman, R., Bernhardt, J., Slaton, N., Blocker, M., Tolbert, A., and Branson, J. 2002. Arkansas Rice Performance Trials, 2000-2002. University of Arkansas Cooperative Extension Service Rice Information Sheet No. 151.

23. Wolpert, T. J., Dunkle, L. D., and Ciuffetti, L. M. 2002. Host-selective toxins and avirulence determinants: What's in a name? Annu. Rev. Phytopathol. 40:251-285.

24. Wrather, J. A., Beck, B., Guethle, D. R., and Cloud, G. 1993. MP646, Rice sheath blight control. Published online by University of Missouri Extension. 